\title{
SÉANCE DU 24 OCTOBRE 1913
}

\author{
PRÉSIDENCE DE M. G. CHAUVEAUD.
}

M. F. Camus, secrétaire, donne lecture du procès-verbal de la dernière séance, dont la rédaction est adoptée.

Par suite de la présentation faite dans la dernière séance, M. le Président proclame membre de la Société :

M. Boıssy (Jean), préparateur à l'École supérieure de Pharmacie de Paris, boulevard d'Argenson, 32 bis,

à Neuilly-sur-Seine, présenté par MM. Guignard et Souèges.

M. le Secrétaire général annonce qu'il a reçu du ministère de l'Instruction publique le programme du Congrès des Sociétés savantes à Paris en 1914. Il donne connaissance des parties de ce programme intéressant la Botanique.

\section{Les propagules des Céphaloziellacées et de quelques autres Hépatiques}

(Suite et fin) ';

PAR M. Ch. DOUIN.

\section{V. - Causes qui influent sur la production DES PROPAGULES.}

D'une façon générale, quand les conditions deviennent mauvaises pour la plante, celle-ci, pour ne pas périr, se met aussitòt à produire des propagules. Les deux causes qui déterminent cette production sont la sécheresse et l'humidité. Une humidité persistante produit le mème effet qu'une sécheresse continue, ce qui prouve une fois de plus que les extrèmes se touchent. Et cette production de propagules est tellement instinctive chez beaucoup d'Hépatiques qu'elle se produit presque au début de la

1. Voir plus haut, p. 477. 


\section{$2 \mathrm{BHL}$ Biodiversity Heritage Library}

1913. "Séance Du 24 Octobre 1913." Bulletin de la Société botanique de France 60, 485-485. https://doi.org/10.1080/00378941.1913.10836659.

View This Item Online: https://www.biodiversitylibrary.org/item/8682

DOI: https://doi.org/10.1080/00378941.1913.10836659

Permalink: https://www.biodiversitylibrary.org/partpdf/160959

\section{Holding Institution}

Missouri Botanical Garden, Peter H. Raven Library

\section{Sponsored by}

Missouri Botanical Garden

\section{Copyright \& Reuse}

Copyright Status: Public domain. The BHL considers that this work is no longer under copyright protection.

This document was created from content at the Biodiversity Heritage Library, the world's largest open access digital library for biodiversity literature and archives. Visit BHL at https://www.biodiversitylibrary.org. 